\title{
Estudio Anatómico de los Arcos Palmares: Diámetro y Presentación
}

\author{
Anatomic Study of the Palmar Archs: Diameter and Presentation \\ "Elizondo-Omaña Rodrigo Enrique; "García-Rodríguez Maria de los Angeles; "Tijerina de la Garza Octavio; \\ "Guzmán López Santos; "De la Garza Castro Oscar; *'López Serna Norberto \& ${ }^{* * *}$ Ortegón Galindo Edgar
}

ELIZONDO-OMAÑA, R. E.; GARCÍA-RODRÍGUEZ, M. A.; TIJERINA, G, O.;GUZMÁN, L. S.; DE LA GARZA, C. O.; LÓPEZ, S. N. \& ORTEGÓN, G. E. Estudio anatómico de los arcos palmares: diámetro y presentación. Int. J. Morphol., 25(1):55-60, 2007.

RESUMEN: Las arterias más importantes de la mano son la radial y ulnar, las que forman los arcos palmares superficial y profundo. La descripción detallada de las características anatómicas de los arcos palmares ha contribuido al desarrollo de nuevas técnicas microquirúrgicas en la mano. Los objetivos de este estudio fueron establecer el patrón arterial de la mano, así como determinar el diámetro luminal de las arterias que contribuyen a la formación de los arcos.

Se realizó un estudio descriptivo, utilizándose 25 manos de cadáveres embalsamados. Se seccionaron las arterias en diferentes puntos para su tinción y observación al microscopio de luz.

El arco palmar superficial completo fue observado en 14 (56\%) muestras y en 11 (44\%) se presentó de manera incompleta. El arco palmar profundo completo se encontró en 20 muestras (80\%) y sólo en 5 (20\%) fue incompleto. La presentación de los arcos arteriales palmares es muy compleja y variada, encontrándose siempre, al menos uno de los arcos completo.

PALABRAS CLAVE: Anatomía; Arco palmar superficial; Arco palmar profundo.

\section{INTRODUCCIÓN}

La irrigación arterial de la mano está dada por ramas de las arterias radial y ulnar, las cuales forman cuatro arcos en la porción distal del miembro superior (Moore, 1999). Los arcos anterior y posterior se forman a nivel del carpo y los arcos superficial y profundo se forman en la palma (Ruengsakulrach et al., 2001). Las clasificaciones propuestas para los arcos palmares son importantes desde el punto de vista quirúrgico (Guzmán \& Castro, 2004; Ruengsakulrach et al.; Patnaik et al., 2002). Estas clasificaciones se basan en estudios anatómicos, para los cuales se han empleado diferentes métodos, como disección macroscópica (Coleman \& Anson, 1961; Ruengsakulrach et al.), inyección intraarterial de látex y silicona (Gellman et al. 2001; Guzmán \& Castro), angiografía (Karlsson \& Niechajev, 1982; Rauch et al., 1999) y ultrasonografía doppler (Al-turk \& Metcalf, 1984). Algunos de estos estudios reportan las variaciones en los arcos arteriales, para lo cual consideran la participación de dife- rentes ramas en su formación (Patnaik et al.), y el número de ramas que éstos emiten (Gellman et al.). Muy pocos estudios reportan el diámetro de los arcos palmares y de sus ramas (Fazan et al. 2004; Gellman et al.).

La descripción detallada de las características anatómicas de los arcos arteriales palmares y de sus diámetros luminales han contribuido a los avances en técnicas microquirúrgicas de la mano (Gellman et al.). El diámetro arterial es un indicador del flujo sanguíneo y es importante para asegurar que existe una adecuada reperfusión de estructuras locales durante una cirugía de reconstrucción de la mano (Fazan et al.). Los diámetros han sido determinados con diferentes técnicas, entre ellas, la medición con caliper electrónico digital (Fazan et al.) y la medición con vernier caliper de un molde intraluminal de látex (Gellman et al.).

\footnotetext{
* Departamento de Anatomía Humana, Facultad de Medicina, UANL. México.

*** Departamento de Anatomía del Desarrollo, Facultad de Medicina, UANL. México.

**** Departamento de Introducción a la Clínica, adscrito a la consulta \#1 del Hospital Universitario Dr. Jose Eleuterio González.

Dirección: Av. Madero y Dr. Aguirre Pequeño s/n, col. Mitras Centro, Monterrey, N. L. 64460, México.
} 
Los objetivos del presente estudio fueron determinar la frecuencia de las variaciones en los patrones arteriales de los arcos palmares superficial y profundo y determinar mediante microscopía de luz, el diámetro luminal de las arterias que forman los arcos y de algunas de sus ramas.

\section{MATERIAL Y MÉTODO}

Se realizó un estudio descriptivo transversal, para el cual se disecaron 25 manos de cadáveres embalsamados (fijados con formol al 10\%), proporcionados por el anfiteatro del Hospital Universitario "Dr. José E. González", Monterrey, México. Se incluyeron todos los especímenes que se encontraran en buen estado de conservación, sin considerar el género ni el lado de la mano (derecho o izquierdo). El procedimiento se realizó en 3 etapas: 1) disección macroscópica, 2) extracción de los arcos y conservación, 3) técnica histológica y mediciones.

Durante la disección macroscópica se documentó por escrito y con fotografía la presentación (completo/incompleto) y tipo (superficial/profundo) de los arcos palmares (según la clasificación presentada por Ruengsakulrach (2001). Para extraer los arcos se realizaron cortes sobre la arteria ulnar inmediatamente proximal al hueso pisiforme, en la rama palmar

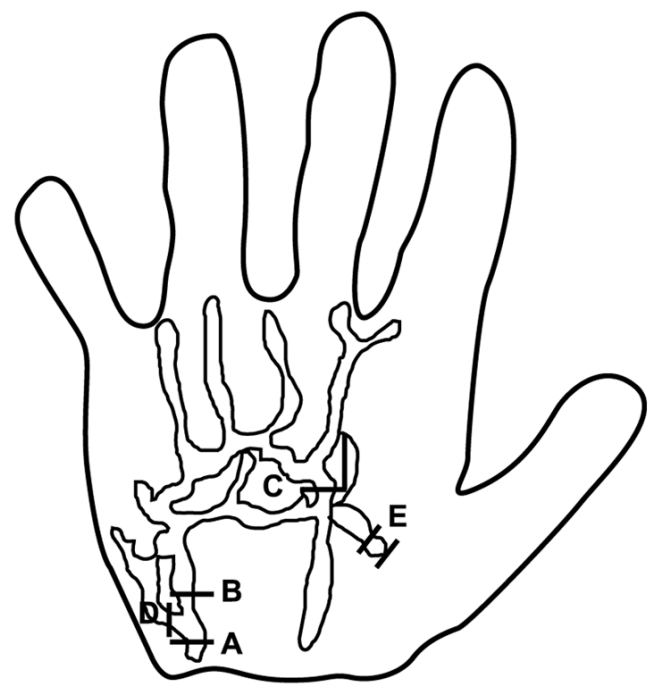

Fig. 1. Muestra los puntos en que se realizaron los cortes en las arterias componentes de los arcos palmares para la obtención de sus diámetros. A) Arteria ulnar a nivel del polo superior del hueso pisiforme; B) arco palmar superficial inmediatamente distal a la división de la arteria ulnar; C) Ramo palmar superficial de la arteria radial en la unión con la (PA1) con la arteria ulnar; D) Rama palmar profunda a nivel su origen; E) Rama palmar profunda de la arteria ulnar a nivel de su punto anastomótico (PA2) con la arteria radial. superficial de la arteria radial a su paso entre los fascículos del músculo aductor del pulgar, y en el arco palmar profundo cuando perfora el primer músculo interóseo dorsal; posteriormente, se identificaron las arterias y se colocaron en solución de formol de $10 \%$. Se realizaron cortes en diferentes puntos de las arterias (Fig. 1) los cuales fueron procesados con la técnica histológica y teñidos con hematoxilina y eosina para su observación al microscopio de luz marca Carl Zeiss ${ }^{\circledR}$. Los diámetros fueron determinados por morfometría y los resultados colocados en tablas utilizando el programa Microsoft Excell 2003®. Se aplicó estadística descriptiva para su interpretación.

Para la clasificación de los arcos palmares se consideraron las siguientes definiciones: el arco palmar superficial se definió como la continuidad entre las arterias ulnar y rama radiopalmar superficial (rama de la arteria radial). Se consideró arco superficial completo tipo 1 cuando existía anastomosis con la rama palmar superficial, y tipo 2 cuando no hay anastomosis con la rama palmar superficial, pero la arteria ulnar irriga el lado medial del pulgar. El arco palmar profundo se definió como la continuidad entre la arteria radial y rama palmar profunda (rama de la arteria ulnar).

\section{RESULTADOS}

Se disecaron 25 manos (16 izquierdas y 9 derechas) con una técnica que permite la obtención de los arcos arteriales de forma completa. Para el análisis estadístico no se consideraron el lado de la mano, ni el sexo del cadáver.

Arco palmar superficial. El arco arterial palmar superficial completo se encontró en 56\% (14/25) de las muestras y en $44 \%$ (11/25) de los casos el arco fue incompleto (Tabla I). El tipo 1 de arco superficial completo se presentó en 10 casos, mientras que el tipo 2 se encontró en 4 casos.

Tabla I. Arcos palmares superficial y profundo, completos e incompletos en 25 manos de cadáveres humanos, formolizados.

\begin{tabular}{lccc}
\hline Arco palmar & Completo & Incompleto & Total \\
Superficial & 14 & 11 & 25 \\
Profundo & 20 & 5 & 25 \\
\hline
\end{tabular}

El diámetro de las arterias que participaron en el arco palmar superficial se muestra en la Tabla II. En algunas manos no fue posible obtener los diámetros de todas las arterias debido a la variedad de arco presentado.

Arco palmar profundo. El arco arterial palmar profundo completo se encontró en el 80\% (20/25) de las manos y en 20\% (5/ 
25) de los casos el arco fue incompleto (Tabla I). El diámetro de las arterias que participan en éste arco se muestra en la Tabla III.

Tabla II. Media y desviación estándar de los diámetros en las arterias que participan en la formación del arco palmar superficial en 25 manos de cadáveres humanos, formolizados.

Diáme tros de las arterias de los arcos palmares superficiales

$\begin{array}{ccc}\text { A } & \text { B } & \text { C } \\ 25 & 24 & 12 \\ 1.87 & 1.80 & 0.83 \\ 0.38 & 0.45 & 0.25 \\ 2.52 & 2.74 & 1.16 \\ 1.12 & 0.86 & 0.18\end{array}$

A) Arteria ulnar a nivel del hueso pisiforme; B) Arco palmar superficial inmediatamente distal a la división de la arteria ulnar; C) Rama palmar superficial de la arteria radial en la unión con la arteria ulnar.

Tabla III. Media y desviación estándar de los diámetros en las arterias que participan en la formación del arco palmar profundo en 25 manos de cadáveres humanos, formolizados.

\begin{tabular}{lcc}
\hline \multicolumn{3}{c}{ Diámetros de los arcos palmares profundos } \\
& D & E \\
& 21 & 17 \\
Media (mm) & 0.97 & 0.75 \\
Desviación estándar & 0.20 & 0.16 \\
Límite superior (mm) & 1.31 & 1.06 \\
Límite inferior (mm) & 0.61 & 0.51 \\
\hline
\end{tabular}

D) Rama palmar profunda a nivel su origen; E) Rama palmar profunda de la arteria ulnar a nivel de su punto anastomótico (PA2) con la arteria radial.

En ninguna de las manos disecadas se presentaron ambos arcos palmares incompletos, es decir, al menos uno de los arcos era completo.

\section{DISCUSIÓN}

Presentación de los arcos palmares. En nuestro estudio la presentación de arco arterial palmar más frecuentemente encontrado fue el completo (tanto superficial como profundo). Esto coincide con lo reportado por Coleman \& Anson; Ikeda et al. (1998); Mezzogiorno et al. (1994; Ruengsakulrach et al. y Gellman et al. Sin embargo, existe una amplia diferencia entre las frecuencias reportadas por estos autores.

Nuestros hallazgos se basaron en la clasificación de arcos palmares, propuesta por Ruengsakulrach et al. y coinciden con lo reportado por dicho autor. En su estudio de 50 manos, encontró en 66\% (33/50) un arco arterial palmar superficial completo y en el restante 34\% (17/50) un arco pal- mar superficial incompleto. Sin embargo, en otro estudio realizado por Ikeda et al., en el que se disecaron 220 manos de cadáveres, se reportó $96.4 \%$ para el arco palmar superficial completo, la cual es muy superior a la reportada en nuestro estudio en el que encontramos una prevalencia del arco arterial palmar superficial completo de 56\% (14/25) y de 44\% (11/25) para el incompleto.

En nuestro estudio la presentación de arco palmar superficial y profundo más frecuentemente encontrado fue el completo y en ningún caso se encontraron ambos arcos incompletos en una misma mano, por lo que, desde un punto de vista funcional, siempre existe circulación colateral en esta región. Esto coincide con lo reportado por varios autores entre ellos Ruengsakulrach et al. y Bianchi (2001); según este último, en los casos en que el arco palmar superficial es incompleto en el lado radial, las colaterales de los dedos correspondientes son emitidas por el arco palmar profundo y por la primera arteria interósea dorsal. Lo anterior confirma que, al menos basándose en criterios anatómicos, es seguro extraer la arteria radial a nivel del antebrazo para usarla en distintos procedimientos quirúrgicos, por ejemplo, como conducto en la cirugía de revascularización miocárdica (Dumanian et al., 1998). Sin embargo, además de que el circuito arterial palmar debe ser anatómicamente completo, el adecuado flujo sanguíneo por la arteria ulnar es un requisito para la extracción de la arteria radial (Brodman et al., 2002).

Autores como Olave et al. (1997); Ruengsakulrach y Mestdagh (1980) reportan la contribución de la arteria mediana, rama de la arteria interósea anterior, al circuito arterial de la mano. Patnaik et al. reportan incluso arcos palmares superficiales dobles (proximal y distal) en 6\% de las manos disecadas (3/50), siendo éstos, a su vez, de dos variedades: arcos radio-ulnares y arcos mediano-ulnares. En nuestro estudio no se encontraron arcos supernumerarios ni contribución de la arteria mediana en ninguna de las manos disecadas.

Diámetros de los arcos palmares. Para la medición de los diámetros de las arterias de la mano se han empleado diferentes métodos, tales como: 1) Ultrasonografía (Hosono et al., 2000; Al-turk \& Metcalf; Doscher et al., 1983; Brodman et al.); 2) Radiográficos (Riekkinen et al., 2003); 3) Macroscópicos simples (Fazan et al.) y 4) Inyección de látex (Gellman et al.; Olave \& Prates, 1999; Haerle et al., 2003).

El estudio de Doscher et al. determina el diámetro de las arterias radial y ulnar por medio de ultrasonografía doppler. Mas recientemente, Hosono et al. utilizando éste método, reporta el diámetro en el antebrazo de la arteria ulnar a tres niveles: proximal, medio y distal; éste último con un diámetro de $2.4 \mathrm{~mm}$ ( $\mathrm{SD}=0.4)$. Brodman et al. determinaron mediante color flow y pulsed Doppler scanning el diámetro luminal y la 
ELIZONDO-OMAÑA, R. E.; GARCÍA-RODRÍGUEZ, M. A.; TIJERINA, G, O.;GUZMÁN, L. S.; DE LA GARZA, C. O.; LÓPEZ, S. N. \& ORTEGÓN, G. E.

Tabla IV. Estudios que reportan la prevalencia de los arcos arteriales palmares.

\begin{tabular}{lcccc}
\hline & \multicolumn{2}{c}{ Arco palmar superficial } & \multicolumn{2}{c}{ Arco palmar profundo } \\
& Incompleto $\%$ & Completo $\%$ & Incompleto $\%$ & Completo $\%$ \\
Coleman \& Anson, 1961 & 21,5 & 78,5 & 3,0 & 97,0 \\
Ikeda et al., 1988 & 3,60 & 96,4 & 23,1 & 76,9 \\
Mezzogiorno et al., 1994 & - & - & 33,3 & 66,7 \\
Rauch et al., 1999 & & 31,8 & & 84,8 \\
Ruengsakulrach et al., 2001 & 34,0 & 66,0 & 10,0 & 90,0 \\
Gellman et al., 2001 & 15,5 & 84,4 & 66,6 & 44,4 \\
Patnaik et al., 2002 & 16,0 & Sencillo 78 & 2,0 & 98,0 \\
Estudio actual & 44,0 & 56,0 & 20,0 & 80,0 \\
\hline
\end{tabular}

velocidad de flujo de las arterias radial y ulnar a nivel de la muñeca en 46 pacientes con enfermedad coronaria, encontrando diámetros promedio de 4,29 $\mathrm{mm}$ y 4,01 $\mathrm{mm}$, respectivamente. Una limitante de este método es que sólo permite evaluar efectivamente el arco palmar superficial (Gellman et al.).

El estudio de Riekkinen et al. que determina el diámetro de las arterias radial y ulnar a nivel de la muñeca por medio de radiografías obtenidas en cadáveres, a los cuales se les inyectó medio de contraste, reportan un diámetro medio para la arteria radial de $3.2 \mathrm{~mm}$ en el antebrazo derecho y de $3 \mathrm{~mm}$ en el antebrazo izquierdo, mientras los diámetros para la arteria ulnar en el antebrazo derecho fueron de 2,5 $\mathrm{mm}$ y en el antebrazo izquierdo de $2,4 \mathrm{~mm}$. Una limitante del estudio es que el diámetro puede estar ligeramente aumentado por efecto de la proyección de la radiografía y otro es el empleo de una regla simple para determinar el diámetro en lugar de un instrumento mas preciso.

Los estudios de Fazan et al. y Gellman et al. reportan el diámetro de la arteria ulnar, la arteria radial, arcos palmares superficial y profundo. En nuestro estudio reportamos los diámetros de las arterias que contribuyen a formar los arcos: arteria ulnar y rama palmar superficial de la arteria radial; rama palmar profunda de la arteria ulnar en su origen y en el sitio de anastomosis con la arteria radial . En nuestro estudio no se estudió el diámetro de la arteria radial y en su lugar se reportó el diámetro de la rama palmar profunda en su anastomosis con la arteria radial al considerar este punto como el inicio del arco arterial palmar profundo.

Los estudios macroscópicos simples (Fazan et al.) y con inyección de látex (Gellman et al.) tal vez sean hasta hoy los más precisos en la determinación del diámetro luminal, al utilizar vernier electrónico y digital. La morfometría es una técnica empleada para determinar mediciones en el microscopio de luz por medio de una reglilla adaptada al ocular, lo que permite tener una mayor precisión al realizar mediciones en una muestra. Esta técnica fue utilizada en nuestro estudio.

Estudios anatómicos que reportan el diámetro de los arcos palmares. La diferencia en diámetro observada entre los estudios (Tabla V) puede ser debida a una retracción que sufre el tejido al ser procesado con la técnica histológica, pero también a una diferencia en la precisión del método empleado para determinar el diámetro. En el estudio de Gellman et al. donde se inyecta látex a presión manual dentro de la arteria, es posible que existiera un cierto grado de dilatación extra secundario a la presión ejercida para introducir el látex.

El empleo de estos conductos para procedimientos de revascularización miocárdica y el uso de técnicas microquirúrgicas de reconstrucción de la mano, requieren de un mejor entendimiento de las presentaciones vasculares y los diámetros luminales de los vasos sanguíneos.

La presentación de los arcos arteriales palmares es muy compleja y variada, encontrándose siempre, al menos uno de los arcos completos. Los diámetros de las arterias que contribuyen a formar estos arcos han sido poco estudiados y existen amplias diferencias entre los resultados de la literatura.

Puesto que existe una amplia diferencia en los diámetros arteriales reportados entre los estudios anatómicos y clínicos, creemos que es importante establecer una comparación entre ellos para tener un factor que proporcione la relación entre los diámetros obtenidos por métodos clínicos y anatómicos.

Tabla V. Diámetros de las arterias ulnar y radial, en manos izquierdas, obtenidos con distintos métodos y por diferentes autores.

\begin{tabular}{lccc}
\hline & Hosono et al., 2000 & Brodman et al., 2002 & Riekkinen et al., 2003 \\
Arteria ulnar & $2.4 \pm 0.4 \mathrm{~mm}$ & $4.01 \mathrm{~mm}$ & $2.5-2.4 \mathrm{~mm}^{*}$ \\
Arteria radial & -- & $4.29 \pm 1.22 \mathrm{~mm}$ & $3.2-3 \mathrm{~mm}^{*}$ \\
\hline
\end{tabular}


ELIZONDO-OMAÑA, R. E.; GARCÍA-RODRÍGUEZ, M. A.; TIJERINA, G, O.;GUZMÁN, L. S.; DE LA GARZA, C. O.; LÓPEZ, S. N. \& ORTEGÓN, G. E. Anatomic study of the palmar archs. Diameter and presentations. Int. J. Morphol., 25(1):55-60, 2007.

SUMMARY: The radial artery and unlar artery are the most important arteries of the hand, they form a superficial and deep palmar arches. The detailed description of the anatomic characteristics of the palmar arches have contributed to develop new microsurgical techniques for the hand. The aim of this study was to establish the arterial pattern in the hand, and also to determine the luminal diameter of the arteries that contributes to palmar arches.

It was a descriptive study. We used 25 hands of embalmed cadavers. We cut the arteries in differents points and after that they were stained and observed in optical microscope. The complete superficial palmar arch was found in 14 (56\%) samples and 11 (44\%) samples it was incomplete. The complete deep palmar arch was found in 20 (80\%) samples and 5 (20\%) it was incomplete. The presentation of the palmar arches is complex and variable, but always exist one complete arch (deep or superficial).

KEY WORDS: Anatomy; Superficial palmar branch; Deep palmar branch.

\section{REFERENCIAS BIBLIOGRÁFICAS}

Al-turk, M. \& Metcalf, W. K. A study of the superficial palmar arteries using the Doppler ultrasonic flowmeter. J. Anat., 138:27-32, 1984.

Bianchi, H. Anatomy of the radial branches of the palmar arch. Variations and its Surgical Importance. Hand Clinics, 19:139-46, 2001.

Brodman, R. F.; Hirsh, L. E. \& Frame R. Effect of radial artery harvest on collateral forearm blood flow and digital perfusion. J. Thorac. Cardiovasc. Surg., 123(3):512-6, 2002.

Coleman, S. S. \& Anson, B. J. Arterial patterns in the hand based upon a study of 650 specimens. Surg. Gynecol. Obstet., 113:409-24, 1961.

Doscher, W.; Viswanathan, B.; Stein, T. \& Margolis, I. Hemodynamic assessment of the circulation in 200 normal hands. Ann Surg., 198(6):776-9, 1983.

Dumanian, G. A.; Segalman, K.; Mispireta, L. A.; Walsh, J. A.; Hendrickson, M. F. \& Wilgis, E. F. Radial artery use in bypass grafting does not change digital blood flow or hand function. Ann. Thorac. Surg., 65(5):12847, 1998 .

Fazan, V. P. S.; Teixeira, B. C.; Hilario, S. J. et al. Superficial Palmar arch: an arterial diameter study. J. Anat., 204:307-11, 2004.

Gellman, H.; Botte, M. J.; Shankwiler, J. et al. Arterial Patterns of the deep and superficial palmar arches. Clin. orthop. rel. research, 383:41-6, 2001.
Guzmán, L. S. \& Castro, G. Y. Variantes del arco arterial palmar superficial. Estandarización del concepto de arcus: un estudio anatómico en la población mexicana. Medicina Universitaria, 6(24):188-93, 2004.

Haerle, M.; Hans-Martin, H.; Klaus, D. et al. Vascular dominance in the forearm. Plast. Reconstr. Surg., 111:1891-8, 2003.

Hosono, M.; Suehiro, S.; Shibata, T. et al. Duplex scanning to assess radial artery suitability for coronary artery bypass grafting. J. Thorac. Cardiovasc. Surg., 48(4): 21721, 2000.

Ikeda, A.; Ogawa, A.; Kazihara, Y.; \& Hamada, N. Arterial patterns in the hand based on a three-dimensional analysis of 220 cadaver hands. J. Hand Surg. (Am.), 13(4):501-9, 1988.

Karlsson, S. \& Niechajev, I. A. Arterial anatomy of the upper extremity. Acta Radiol Diag., 23:115-21, 1982.

Mezzogiorno, A.; Passiatore, C. \& Mezzogiorno, V. Anatomic variations of the deep palmar arteries in man. Acta Anat., 149(3):221-4, 1994.

Mestdagh, H. Anatomy of the arcus volaris profundus of man (author's transl). Anat Anz., 147(2):180-7, 1980.

Moore, K. L. Anatomía con orientación clínica. $3^{\text {a }}$ ed.Madrid, Editorial Médica Panamericana, 1999. pp. 794-5.

Olave, E. \& Prates, J. C. Deep palmar arch patterns in Brazilian individuals. Surg. Radiol. Anat., 21(4):267-71, 1999. 
Olave, E.; Prates, J. C.; Gabrielli, C. \& Pardi, P. Median artery and superficial palmar branch of the radial artery in the carpal tunnel. Scand. J. Plast. Reconstr. Surg Hand Surg., 31:13-6, 1997.

Patnaik, V. V. G.; Kalsey, G. \& Singla, R. K. Palmar arterial arches a morphological study. J. Anat. Soc. India. 51(2):187-93, 2002.

Rauch, D.; Fischer, C.; Achenbach, S.; Klose, K. J. \& Wagner, H. J. Angiography detection of closed palmar arcs. Rofo., 171(3):207-10, 1999.

Riekkinen, H. V.; Karkola, K. O. \& Kankainen, A. The radial artery is larger than the ulnar. Ann. Thorac. Surg., 75:882-4, 2003.

Ruengsakulrach, P.; Eizenberg, N.; Fahrer, C. et al. Surgical implications of variations in hand collateral circulation: anatomy revisited. J. Thorac. Cardiovasc. Surg., 122:682-6, 2001.
Dirección para correspondencia:

Dr. Rodrigo Enrique Elizondo-Omaña M.D.

Department of Human Anatomy.

Medicine School. Col. Mitras Centro,

C.P. 64460

Monterrey, Nuevo León.

MÉXICO

E-mail: rod_omana@yahoo.com

Recibido : 06-10-2006

Aceptado: 20-12-2006 